\title{
Editorial
}

\section{Dynamical Aspects of Initial/Boundary Value Problems for Ordinary Differential Equations 2014}

\author{
Jifeng Chu, ${ }^{1}$ Yongli Song, ${ }^{2}$ Juntao Sun, ${ }^{3}$ Patricia J. Y. Wong, ${ }^{4}$ and Yonghui Xia ${ }^{5}$ \\ ${ }^{1}$ Department of Mathematics, College of Science, Hohai University, Nanjing 210098, China \\ ${ }^{2}$ Department of Mathematics, Tongji University, Shanghai 200092, China \\ ${ }^{3}$ School of Science, Shandong University of Technology, Zibo, Shandong 255049, China \\ ${ }^{4}$ School of Electrical and Electronic Engineering, Nanyang Technological University, 50 Nanyang Avenue, Singapore 639798 \\ ${ }^{5}$ Department of Mathematics, Zhejiang Normal University, Jinhua 321001, China
}

Correspondence should be addressed to Jifeng Chu; jifengchu@126.com

Received 28 September 2014; Accepted 28 September 2014

Copyright (C) 2015 Jifeng Chu et al. This is an open access article distributed under the Creative Commons Attribution License, which permits unrestricted use, distribution, and reproduction in any medium, provided the original work is properly cited.

Dynamical aspects of initial/boundary value problems for ordinary differential equations have become a rapidly growing area of research in the theory of differential equations and dynamical systems and have gathered substantial research interests during the last decades. The attractiveness of this field not only is derived from theoretical interests, but also is motivated by the insights that such dynamical aspects could reveal several phenomena observed in applied sciences.

The current special issue places its emphasis on the study of the dynamical aspects of initial/boundary value problems for ordinary differential equations. Call for papers has been carefully prepared by the guest editors and posted on the journals web page. All manuscripts submitted to this special issue went through a thorough peer-refereeing process. The response to this special issue was beyond our expectation. We received 49 papers in these research fields. Based on the reviewers' reports, we collect twenty-three original research articles. Besides, one survey on recent results for variational approach to impulsive problems is also contained.

It is certainly impossible to provide in this short editorial note a more comprehensive description for all articles in this special issue. However, the team of the guest editors believes that results included reflect some recent trends in research and outline new ideas for future studies of dynamical aspects of initial/boundary value problems for ordinary differential equations.

\section{Acknowledgments}

We would like to express our gratitude to the authors who have submitted papers for consideration. Thanks are also due to the many reviewers whose reports are important for us to make the decisions. All the participants have made it possible to have a very stimulating interchange of ideas. We would also like to thank the editorial board members of this journal, for their support and help throughout the preparation of this special issue.

Jifeng Chu Yongli Song Juntao Sun

Patricia J. Y. Wong Yonghui Xia 


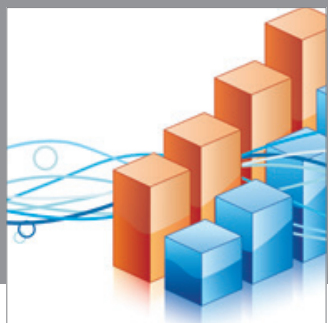

Advances in

Operations Research

mansans

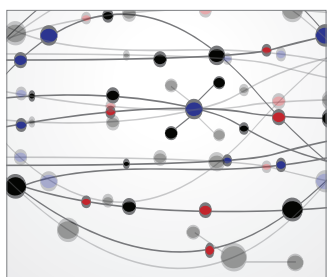

The Scientific World Journal
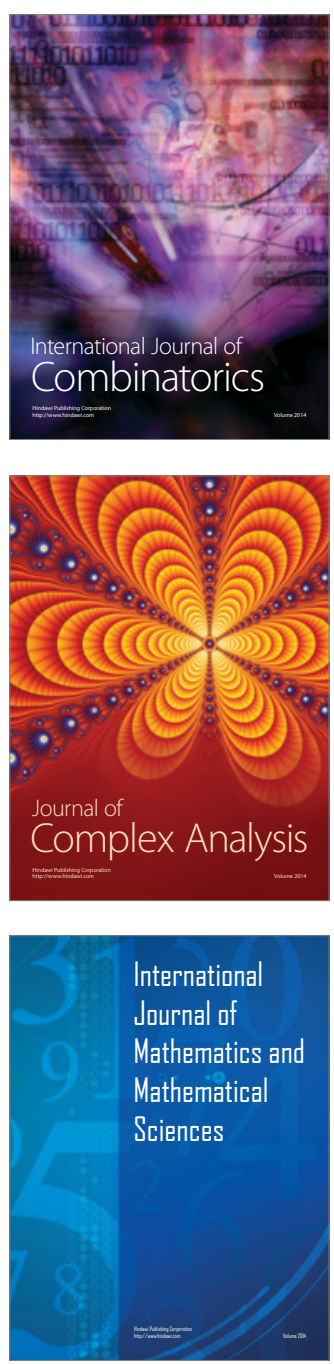
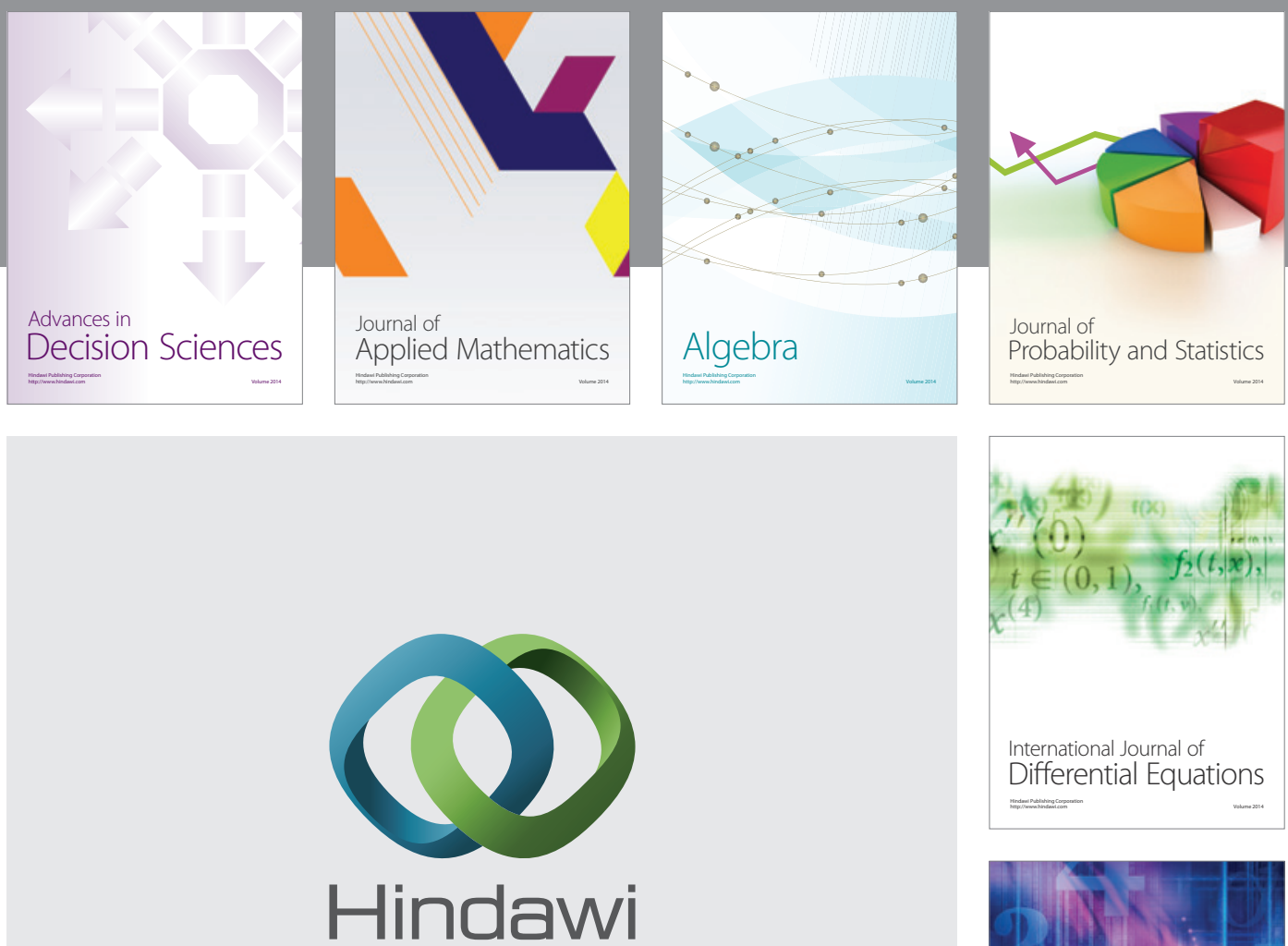

Submit your manuscripts at http://www.hindawi.com
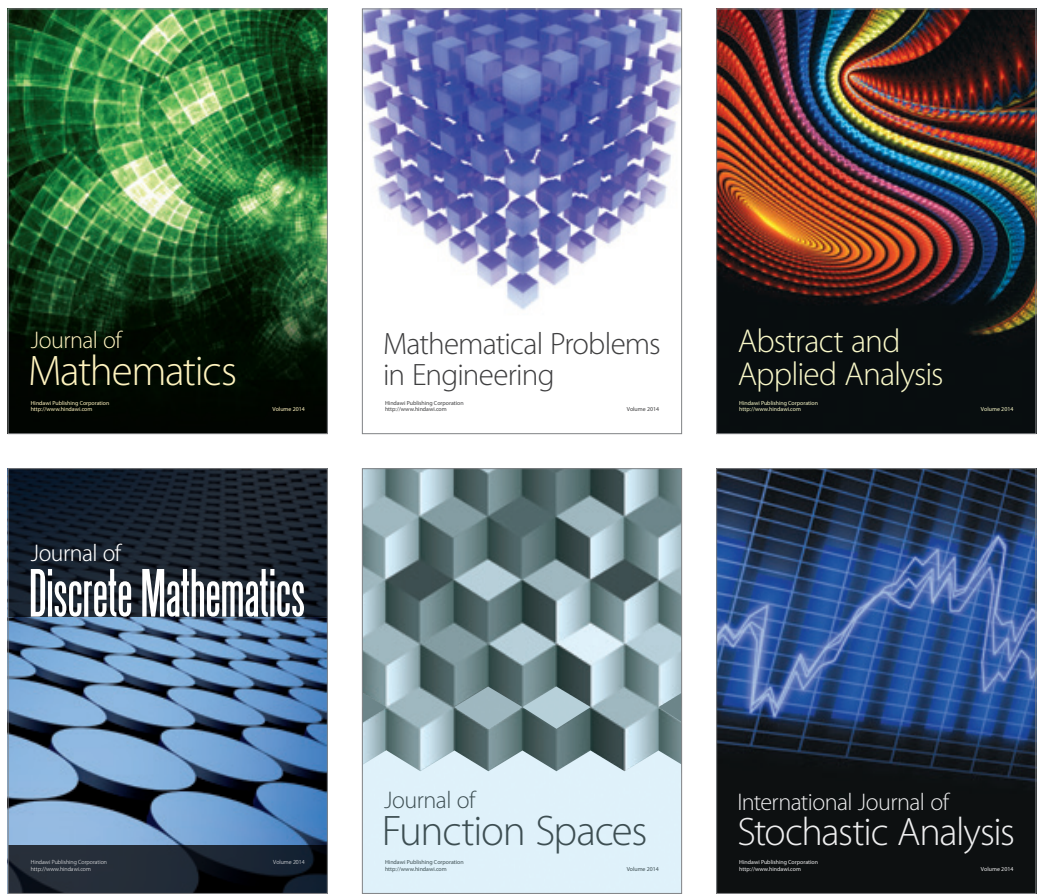

Journal of

Function Spaces

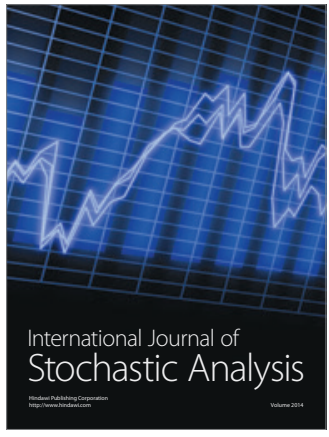

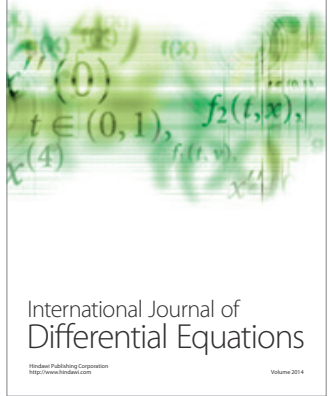
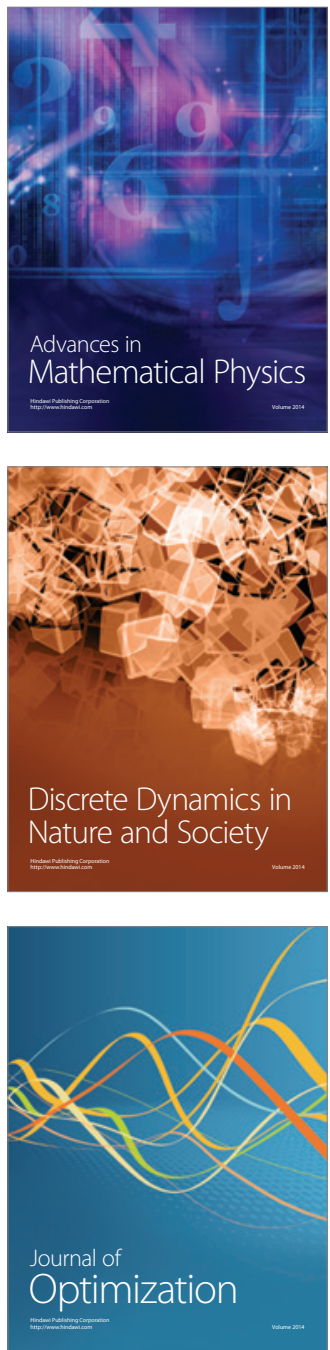